\title{
Relaciones entre conducta agresiva y metas académicas: estudio con una muestra de estudiantes españoles de Educación Secundaria Obligatoria*
}

\section{Relationship between Aggessive Behavior and Academic Goals: Study with a Spanish Compulsory Secondary Education Sample of Students}

Recibido: abril 4 de 2011 | Revisado: noviembre 12 de 2011 | Aceptado: marzo 12 de 2012

\author{
MARÍA S. TORREGROSA ** \\ Universidad Católica San Antonio de Murcia, España \\ CÁNDIDO J. INGLÉS *** \\ Universidad Miguel Hernández de Elche, España \\ José M. GARCÍA-FERNÁNDEZ ***** \\ Universidad de Alicante, España \\ ANTONIO VALLE ****** \\ Universidad de A Coruña, España \\ José C. NúNEZ ******* \\ Universidad de Oviedo, España
}

SICI: 1657-9267(201212)11:4<1303:RECAMA>2.0.TX;2-N

Para citar este artículo: Torregrosa, M. S., Inglés, C. J., García-Fernández, J. M., Valle, A. \& Núñez, J. C. (2012). Relaciones entre conducta agresiva y metas académicas: estudio con una muestra de estudiantes españoles de Educación Secundaria Obligatoria. Universitas Psychologica, 11(4), 1303-1315.

Artículo de investigación financiado a través del Proyecto SEJ 2004-07311/EDUC perteneciente al Plan Nacional de Investigación Científica, Desarrollo e Innovación Tecnológica del Ministerio de Educación y Ciencia concedido al segundo autor.

** Investigador en el Área de Psicología Evolutiva y de la Educación del Dpto. de Educación. E-mail: mstorregrosa@ucam.edu

*** Profesor Titular de Universidad en el Área de Psicología Evolutiva y de la Educación del Dpto. de Psicología de la Salud.E-mail: cjingles@umh. es. ResearcherID: Inglés, C. C-5979-2011.

***** Profesor Titular de Universidad en el Área de Psicología Evolutiva y de la Educación del Dpto. de Psicología Evolutiva y Didáctica. E-mail: josemagf@ua.es

****** Catedrático de Psicología de la Educación en el Dpto. de de Psicología Evolutiva y de la Educación.E-mail: vallar@udc.es

********* Catedrático de Psicología de la Educación en el Área de Psicología Evolutiva y de la Educación del Dpto. de Psicología.E-mail: jcarlosn@uniovi.es

\section{RES U MEN}

Este estudio analizó la relación entre la conducta agresiva y las metas académicas en una muestra de 2.022 estudiantes españoles de Educación Secundaria Obligatoria (ESO). La conducta agresiva fue evaluada con el Teenage Inventory of Social Skills (TISS) y las metas académicas mediante el Achievement Goal Tendencies Questionnaire (AGTQ). Los resultados revelaron que los estudiantes con alta conducta agresiva, de ambos sexos y de todos los cursos académicos de ESO, presentaron puntuaciones significativamente más altas en metas de reforzamiento social que sus iguales con baja conducta agresiva. Los análisis de regresión logística mostraron que la conducta agresiva fue un predictor positivo y estadísticamente significativo de las metas de reforzamiento social en ambos sexos y en todos los cursos de ESO.

Palabras clave autores

Adolescencia, conducta agresiva, educación secundaria, metas académicas.

Palabras clave descriptores

Conflicto, educación meta, España.

\section{A B S T R A C T}

This study analyzed the relationship between aggressive behavior and academic goals in a sample of 2.022 Spanish students of Compulsory Secondary Education (CSE). Aggressive behavior was assessed with the Teenage Inventory of Social Skills (TISS), and academic goals were assessed with the Achievement Goal Tendencies Questionnaire (AGTQ). The results revealed that students with high aggressive behavior, in both sexes and for all academic grades of CSE, had significantly higher scores in social reinforcement goals than their peers with low aggressive behavior. Logistic regression analyses showed that high aggressive behavior was a positive and statistically significant predictor of high social reinforcement goals, in both sexes and for all academic grades of CSE.

Key words authors

Adolescence, Aggressive Behavior, Secondary Education, Academic Goals.

Key words plus

Conflict, Education Goals, Spain. 
El estudio sobre las metas que orientan la actuación de los adolescentes ha sido ampliamente abordado en los últimos años, destacando la importancia concedida a las metas académicas en este periodo del ciclo vital (e.g., López-Romero \& Romero, 2010; Massey, Gebhardt \& Garnefski, 2008; Valle, Rodríguez et al., 2009). Sin embargo, los estudios que analizan la relación entre conducta agresiva y metas académicas en estudiantes de Educación Secundaria son aún escasos a nivel internacional (Gilman \& Anderman, 2006; Little \& Garber, 2004; Wentzel \& Asher, 1995), y prácticamente inexistentes en España (LópezRomero \& Romero, 2010). Esta situación podría considerarse, cuando menos, singular, puesto que ambas variables son consideradas factores clave para el ajuste personal, social y académico de los adolescentes (véanse para revisión, Marcus, 2007; Massey et al., 2008; Meece, Anderman \& Anderman, 2006). Por tanto, el presente estudio pretende contribuir al conocimiento sobre la relación entre el comportamiento agresivo hacia los iguales y las metas académicas en estudiantes españoles de Educación Secundaria Obligatoria (ESO), considerando el comportamiento agresivo como toda aquella conducta que supone la utilización de medios coercitivos para hacer daño a otros iguales de forma puntual y satisfacer los intereses propios (Torregrosa et al., 2012; Trianes, 2000), y las metas académicas como la tendencia de los estudiantes a estudiar para incrementar su competencia (i.e., metas de aprendizaje), avanzar en sus estudios (i.e., metas de logro) y obtener juicios positivos evitando, simultáneamente, valoraciones negativas de sus compañeros de clase, profesores y padres (i.e., metas de reforzamiento social) (Hayamizu \& Weiner, 1991).

\section{Importancia de la conducta agresiva en ESO}

Tradicionalmente, una de las temáticas que más ha preocupado y ocupado a investigadores en Psicología, Educación y otras disciplinas afines, ha sido el estudio de la conducta agresiva en la adolescencia (e.g., Marcus, 2007), debido, entre otras razones, a su elevada prevalencia (e.g., Díaz-Aguado,
Martínez-Arias \& Martín-Seoane, 2004; Inglés et al., 2008) y a sus consecuencias negativas sobre el ajuste psicológico y social de los adolescentes (e.g., Andreu, Peña \& Larroy, 2010; Estévez, Martínez, Herrero \& Musitu, 2006; Estévez, Murgui \& Musitu, 2008; Inglés et al., 2007; Inglés, Delgado, García-Fernández, Ruíz-Esteban \& Díaz-Herrero, 2010; Saab \& Klinger, 2010).

La evidencia empírica previa también ha revelado que la conducta agresiva se relaciona positivamente con un desajuste escolar significativo (e.g., Dodge, Coie \& Lynam, 2006; Marcus, 2007). En este sentido, los estudiantes que se comportan agresivamente presentan frecuentemente bajo rendimiento académico (e.g., Herrenkohl, Catalano, Hemphill \& Toumbourou, 2009; Jimerson \& Ferguson, 2007; Loveland, Lounsbury, Welsh \& Buboltz, 2007; Torregrosa et al., 2012) y abandono prematuro de los estudios (e.g., Farmer et al., 2003). Paralelamente, estos estudiantes suelen caracterizarse por un autoconcepto académico negativo (e.g., Estévez, Martínez \& Musitu, 2006; Marsh, Parada \& Ayotte, 2004), un patrón atribucional desadaptativo (e.g., Torregrosa, Inglés, Delgado \& García-Fernández, 2008), y una selección y uso inadecuados de sus estrategias de aprendizaje (e.g., Rodríguez-Mayoral, Martínez-Arias, Díaz-Aguado \& Morentín, 2008). Todos estos factores pueden contribuir, al menos en parte, a que los jóvenes con elevada agresividad presenten una actitud negativa hacia la escuela y el profesorado (e.g., Estévez, Murgui, Moreno \& Musitu, 2007).

\section{Importancia de la motivación académica en ESO}

El incremento significativo en las tasas de fracaso escolar y abandono prematuro de los estudios por parte de los estudiantes españoles de ESO, ha dado lugar a que ambas cuestiones se conviertan en problemas importantes a los que debe enfrentarse el sistema educativo español (Ministerio de Educación y Ciencia, 2010). Este fenómeno preocupante ha conducido a profesores, psicólogos educativos, pedagogos, psicopedagogos, responsables de la política educativa e investigadores a reconsiderar qué 
factores están implicados en la falta de interés de los estudiantes por las tareas escolares (Pintrich, 2003). En esta línea, diversas investigaciones han indicado que la motivación académica es un factor relevante en la predicción del rendimiento académico (e.g., Inglés et al., 2009; Steinmayr \& Spinath, 2009) y en la prevención del fracaso escolar y el abandono prematuro de los estudios (e.g., Alderman, 2008; Elliot \& Dweck, 2005; Meece et al., 2006; Pintrich, 2003), ya que actúa facilitando la aparición de estrategias de aprendizaje que mejoran el rendimiento académico de los alumnos a través de un análisis más profundo de la información (Covington, 2000; Dina \& Efklides, 2009). Así, un patrón motivacional adaptativo podría favorecer el éxito académico de los estudiantes, incrementando la tasa de graduados en Educación Secundaria (Wigfield \& Eccles, 2002).

\section{Relaciones entre conducta agresiva y motivación académica}

Aunque numerosos investigadores han encontrado que el funcionamiento social y la motivación académica de los estudiantes están intrínsecamente entrelazados (e.g., Wentzel, 2005; Wentzel \& Watkins, 2002), actualmente existen pocos estudios dirigidos a examinar la relación entre conducta agresiva y metas académicas en estudiantes de Educación Secundaria.

Wentzel y Asher (1995) analizaron la relación entre la conducta agresiva informada por los iguales y la motivación escolar valorada por los profesores en una muestra de 423 estudiantes norteamericanos de 11 a 13 años, encontrando que los adolescentes agresivos-rechazados presentaban un menor interés por el trabajo escolar que sus compañeros. En la misma línea, Gilman y Anderman (2006) hallaron, usando una muestra de 654 estudiantes norteamericanos de $9^{\circ}$ grado, que los alumnos identificados por sus iguales como más proclives a comenzar peleas mostraban menor interés por las tareas de aprendizaje y por aumentar su competencia. Finalmente, Little y Garber (2004), en un estudio longitudinal realizado con 129 adolescentes estadounidenses, encontraron relaciones negativas y estadísticamente significativas entre la conducta agresiva (informada por los padres y los propios estudiantes) y la orientación de los estudiantes hacia la consecución de éxitos en el ámbito académico.

En España, López-Romero y Romero (2010) encontraron, usando una muestra de 488 estudiantes de 12 a 18 años, una relación negativa y estadísticamente significativa entre distintas variables relacionadas (e.g., agresión, robo, consumo de drogas, incumplimiento de las normas del grupo, etc.) y la importancia concedida a distintos tipos de metas (e.g., metas académicas, interpersonales, etc.).

\section{El presente estudio}

La revisión de la investigación previa relativa a las relaciones entre conducta agresiva y motivación académica, además de ser escasa, presenta varias limitaciones. En primer lugar, aunque varios estudios han destacado que las manifestaciones agresivas en la adolescencia media tienden a caracterizarse por conductas de tipo verbal o relacional (e.g., Herrero, Estévez \& Musitu, 2006; Santisteban, Alvarado \& Recio, 2007; Toldos, 2005), Gilman y Anderman (2006) evaluaron las conductas agresivas en esta etapa del ciclo vital, atendiendo únicamente a conductas de tipo físico. En segundo lugar, Wentzel y Asher (1995) evaluaron las metas académicas de los estudiantes a partir de la información proporcionada por sus profesores. Sin embargo, tal y como argumentó Dweck (1986), cada estudiante establece, de forma personal y subjetiva, cómo abordar las situaciones para alcanzar el logro académico, por lo que los autoinformes proporcionan información más apropiada para la medida y evaluación de esta variable. En tercer lugar, los estudios que han examinado la relación entre conducta agresiva y motivación escolar únicamente se han centrado en una de las dos orientaciones principales de la motivación (i.e., metas de aprendizaje o metas de rendimiento). Además, ninguno de los estudios previamente realizados ha considerado la distinción entre los dos tipos principales de metas de rendimiento (i.e., metas de logro y metas de reforzamiento social). Este hecho también resulta singular ya que ambos tipos de metas se relacionan 
diferencialmente con el rendimiento escolar y con diversas variables cognitivo-motivacionales, tanto en estudiantes de Educación Secundaria (e.g., Inglés et al., 2009; Valle, Núñez et al., 2009) como en estudiantes de Educación Superior (e.g., Hayamizu \& Weiner, 1991; Valle et al., 2003, 2010). Además, la evidencia empírica previa ha constatado que los adolescentes que se comportan agresivamente conceden una atención especial a la valoración que hacen los demás de ellos (e.g., Ryan \& Shim, 2008; López-Romero \& Romero, 2010), por lo que cabría esperar que presenten una mayor orientación hacia metas de reforzamiento social.

Finalmente, ninguno de los estudios previamente realizados ha tenido en consideración las variables sexo, edad y/o curso académico, aun cuando numerosas investigaciones, realizadas usando muestras de estudiantes de Educación Secundaria, han encontrado diferencias de sexo y edad o curso académico en conducta agresiva (e.g., Andreu et al., 2010; Ang, 2007; Herrero et al., 2006; Inglés, Hidalgo, Méndez \& Inderbitzen, 2003; Inglés et al., 2008; Lahey et al., 2000; Peets \& Kikas, 2006; Santisteban et al., 2007; Toldos, 2005) y metas académicas (e.g., Broc, 2006; Butler, 2006; Castillo, Balaguer \& Duda, 2001; Delgado, Inglés, GarcíaFernández, Castejón \& Valle, 2010; Núñez, MartínAlbo, Navarro \& Suárez , 2010).

Teniendo en cuenta estas limitaciones, el presente estudio tiene un doble objetivo: (a) analizar las diferencias en metas académicas entre estudiantes españoles de ESO con baja y alta conducta agresiva, atendiendo a las variables sexo y curso académico y (b) determinar en qué medida la alta conducta agresiva hacia los iguales pronostica altas metas de aprendizaje, de rendimiento y de reforzamiento social en función del sexo y el curso académico.

A partir de los hallazgos de investigaciones previas, se espera que:

1. Los estudiantes de ambos sexos y de todos los cursos de ESO con alta conducta agresiva presenten, respecto a sus iguales con baja conducta agresiva, metas de aprendizaje y de logro significativamente más bajas.

2. Los estudiantes de ambos sexos y de todos los cursos de ESO con alta conducta agresiva pre- senten metas de reforzamiento social significativamente más altas que sus compañeros con baja conducta agresiva.

3. La conducta agresiva sea un predictor negativo y estadísticamente significativo de las metas de aprendizaje y de rendimiento y un predictor positivo y estadísticamente significativo de las metas de reforzamiento social en los estudiantes de ambos sexos y de todos los cursos de ESO.

\section{Método}

\section{Participantes}

Se realizó un muestreo aleatorio por conglomerados, siendo las unidades primarias de muestreo las zonas geográficas (centro, norte, sur, este y oeste) de dos provincias españolas pertenecientes a dos comunidades autónomas. Las unidades secundarias fueron los centros escolares de cada zona geográfica y, finalmente, las unidades terciarias fueron las aulas. Con el fin de que todas las zonas geográficas estuvieran representadas se seleccionaron aleatoriamente 20 centros de áreas rurales y urbanas, 14 públicos y 6 privados. Cada zona geográfica estuvo representada por una media de dos centros. Una vez determinados los centros del estudio, se seleccionaron aleatoriamente cuatro aulas, computándose aproximadamente 120 sujetos por centro.

El total de sujetos seleccionados fue 2.267, de los que 116 (5.12\%) fueron excluidos por errores $\mathrm{u}$ omisiones en sus respuestas o por no obtener por escrito el consentimiento informado de los padres para participar en la investigación y 129 (5.69\%) fueron excluidos por ser extranjeros con importantes déficits en el dominio de la lengua española.

La muestra final se compuso de 2.022 estudiantes (1.033 hombres y 989 mujeres) de ESO, con un rango de edad de 12 a 16 años $(M=13.81$; $D E=1.35)$. La distribución de la muestra por curso académico fue la siguiente: 1 $\mathrm{ESO}(576 ; 309$ hombres y 267 mujeres), 2을 $\mathrm{ESO}$ (505; 251 hombres y 254 mujeres), 3을 $\mathrm{ESO}$ (502; 260 hombres y 242 mujeres) y 4 o de ESO (439; 213 hombres y 226 
mujeres). La prueba chi cuadrado de homogeneidad de la distribución de frecuencias reveló la ausencia de diferencias estadísticamente significativas entre los ocho grupos de Sexo x Curso $\left(\chi^{2}{ }_{(3,2022)}=3.16\right.$; $p=0.37)$. La composición étnica de la muestra fue la siguiente: $88.9 \%$ españoles, $6.34 \%$ hispanoamericanos, 3.37\% resto de Europa, 0.75\% asiáticos y $0.64 \%$ árabes.

\section{Instrumentos}

Teenage Inventory of Social Skills [TISS] (Inderbitzen $\mathcal{E}$ Foster, 1992)

El TISS evalúa la conducta prosocial y agresiva de los adolescentes en las relaciones con sus iguales. Consta de 40 ítems agrupados en dos escalas: Conducta Prosocial y Conducta Agresiva. Los ítems son valorados mediante una escala Likert de 6 puntos $(1=$ no me describe nada; 6 = me describe totalmente). El TISS ofrece dos puntuaciones, una para conducta prosocial y otra para conducta agresiva, las cuales se obtienen sumando los valores asignados por los sujetos a los 20 ítems que componen cada dimensión. Puntuaciones altas indican elevada conducta prosocial y agresiva.

El TISS es uno de los instrumentos de evaluación de las habilidades sociales con mejores garantías psicométricas en población anglo e hispanoparlante (Inglés, Méndez, Hidalgo, Rosa \& Estévez, 2003). La estructura bifactorial de la versión española del TISS fue confirmada por Inglés, Hidalgo et al. (2003) en una muestra de 660 adolescentes españoles, siendo los coeficientes de consistencia interna (alfa de Cronbach) adecuados en ambos factores: 0.89 (Conducta Prosocial) y 0.84 (Conducta Agresiva). La validez de constructo de las puntuaciones del TISS también fue apoyada mediante correlaciones estadísticamente significativas con puntuaciones de otros cuestionarios de conducta social (asertividad, ansiedad social, sumisión, agresividad y arrogancia) y variables de personalidad (neuroticismo, psicoticismo y extraversión). En el presente estudio se utilizó únicamente la escala de Conducta Agresiva, siendo el coeficiente de consistencia interna (alfa de Cronbach) 0.82.

\section{Achievement Goal Tendencies Questionnaire [AGTQ] (Hayamizu $\& 3$ Weiner, 1991)}

El AGTQ evalúa las orientaciones de meta de los estudiantes en el ámbito escolar. Consta de 20 ítems, agrupados en tres factores: Metas de Aprendizaje (MA; 8 ítems), Metas de Reforzamiento Social (MRS; 6 ítems) y Metas de Logro (ML; 6 ítems). Los ítems son valorados mediante en una escala Likert de 5 puntos $(1=$ Nunca; $5=$ Siempre $)$. Las puntuaciones en las escalas del AGTQ se obtienen sumando los valores asignados por los sujetos a los ítems que componen cada dimensión. Puntuaciones altas indican altas metas de aprendizaje, de logro y de reforzamiento social.

La evidencia de fiabilidad y validez de las puntuaciones de la versión española del AGTQ fue examinada por Inglés et al. (2009) en una muestra de 2.022 estudiantes de ESO. Los resultados de este estudio apoyaron la estructura tridimensional de las puntuaciones del AGTQ, obteniendo, además, otros índices de fiabilidad y validez de constructo satisfactorios. Asimismo, los resultados hallados recientemente por Inglés, et al. (2011) apoyaron la invarianza factorial del modelo tridimensional del AGTQ a través del sexo y la edad, revelando que el significado de los ítems del cuestionario es equivalente en los estudiantes de ambos sexos y de todos los cursos de ESO. Los coeficientes alfa de Cronbach en el presente estudio fueron: 0.8 (MA), 0.77 (MRS) y 0.74 (ML).

\section{Procedimiento}

Se llevó a cabo una entrevista con los directores y psicopedagogos de los centros participantes para exponer los objetivos de la investigación, describir el instrumento de evaluación, solicitar los permisos pertinentes y promover su colaboración. Posteriormente, se celebró una reunión con los padres para explicarles el estudio y solicitar el consentimiento informado, por escrito, autorizando a sus hijos a participar en la investigación. Ambas pruebas fueron contestadas de forma colectiva y anónima en el aula a finales del curso escolar. Los investigadores estuvieron presentes durante la administración de las pruebas para proporcionar ayuda si era necesaria 
y verificar la cumplimentación independiente por parte de los participantes.

\section{Análisis estadísticos}

La identificación de estudiantes con baja y alta conducta agresiva se estableció una vez comprobado que la distribución de las puntuaciones de los participantes del estudio se ajustaba a la distribución teórica esperada en la escala de Conducta Agresiva del TISS. Así, la muestra general $(N=2.022)$ se dividió en dos grupos: (a) sujetos con baja conducta agresiva: puntuaciones iguales o inferiores al centil $25\left(N_{1}=517 ; 25.6 \%\right)$ y; (b) sujetos con alta conducta agresiva: puntuaciones iguales o superiores al centil $75\left(N_{2}=535 ; 26.5 \%\right)$.
Para analizar las diferencias en MA, MRS y ML entre estudiantes con baja y alta conducta agresiva se aplicó la prueba $t$ de Student. Debido al elevado tamaño muestral del estudio, la prueba t puede detectar erróneamente diferencias estadísticamente significativas. Por esta razón se incluye, además, el índice d (diferencia media tipificada) propuesto por Cohen (1988), que permite valorar la magnitud o el tamaño del efecto de las diferencias encontradas (Sun, Pan \& Wang, 2010). Su interpretación es sencilla: $0.20 \leq d \leq 0.50$ supone un tamaño del efecto pequeño, mientras que $0.51 \leq d \leq 0.79$ es moderado y $d \geq 0.80$ es grande.

Con el fin de examinar y cuantificar la capacidad predictiva de la conducta agresiva sobre las metas académicas, se llevaron a cabo análisis de

TABLA 1

Metas académicas en estudiantes con baja y alta conducta agresiva

\begin{tabular}{|c|c|c|c|c|c|c|c|}
\hline \multirow{2}{*}{$\begin{array}{c}\text { Metas académicas (rango) } \\
\text { Hombres }\end{array}$} & \multicolumn{2}{|c|}{ Baja conducta agresiva } & \multicolumn{2}{|c|}{ Alta conducta agresiva } & \multicolumn{3}{|c|}{ Significación estadística } \\
\hline & $M$ & $\mathrm{DE}$ & $M$ & $\mathrm{DE}$ & $t_{536}$ & $p$ & $d$ \\
\hline MA $(8-40)$ & 30.14 & 5.45 & 30.13 & 5.95 & 0.03 & ns & - \\
\hline MRS (6-30) & 19.20 & 5.42 & 21.68 & 5.08 & -5.12 & 0.00 & -0.48 \\
\hline $\operatorname{ML}(6-30)$ & 24.97 & 3.86 & 25.21 & 3.89 & -0.66 & ns & - \\
\hline Mujeres & M & $\mathrm{DE}$ & M & $\mathrm{DE}$ & $t_{536}$ & $p$ & $d$ \\
\hline MA $(8-40)$ & 30.99 & 5.60 & 30.35 & 5.83 & 1.19 & ns & - \\
\hline MRS (6-30) & 19.67 & 5.51 & 21.66 & 5.62 & -3.79 & 0.00 & -0.36 \\
\hline $\operatorname{ML}(6-30)$ & 25.85 & 3.61 & 25.86 & 3.80 & -0.04 & ns & - \\
\hline $1^{\circ} \mathrm{ESO}$ & M & $\mathrm{DE}$ & M & $\mathrm{DE}$ & $t_{536}$ & $p$ & $d$ \\
\hline MA (8-40) & 31.11 & 5.64 & 30.22 & 6.21 & 1.31 & ns & - \\
\hline MRS (6-30) & 20.53 & 4.97 & 21.87 & 5.15 & -2.31 & 0.02 & -0.26 \\
\hline $\operatorname{ML}(6-30)$ & 25.45 & 3.88 & 24.98 & 4.10 & 2.11 & ns & - \\
\hline $2^{\circ} \mathrm{ESO}$ & M & $\mathrm{DE}$ & M & $\mathrm{DE}$ & $t_{536}$ & $p$ & $d$ \\
\hline MA $(8-40)$ & 30.04 & 5.81 & 29.45 & 5.72 & 0.86 & ns & - \\
\hline MRS (6-30) & 19.56 & 5.57 & 21.89 & 4.47 & -3.84 & 0.00 & -0.46 \\
\hline $\operatorname{ML}(6-30)$ & 25.59 & 3.78 & 25.39 & 3.75 & 0.44 & ns & - \\
\hline 3을 $\mathrm{ESO}$ & M & $\mathrm{DE}$ & M & $\mathrm{DE}$ & $t_{536}$ & $p$ & $d$ \\
\hline MA $(8-40)$ & 30.35 & 5.36 & 30.65 & 5.81 & -0.44 & ns & - \\
\hline MRS (6-30) & 18.71 & 5.54 & 21.38 & 5.70 & -3.88 & 0.00 & -0.47 \\
\hline ML (6-30) & 25.31 & 3.62 & 25.74 & 3.71 & -0.98 & $\mathrm{~ns}$ & - \\
\hline $4^{\circ} \mathrm{e}$ ESO & M & $\mathrm{DE}$ & M & $\mathrm{DE}$ & $t_{536}$ & $p$ & $d$ \\
\hline MA $(8-40)$ & 31.52 & 5.23 & 30.56 & 5.83 & 1.22 & ns & - \\
\hline MRS (6-30) & 18.87 & 5.86 & 21.51 & 5.76 & -3.20 & 0.00 & -0.45 \\
\hline ML (6-30) & 26.03 & 3.46 & 26.32 & 3.71 & -0.56 & ns & - \\
\hline
\end{tabular}

Nota $. \mathrm{MA}=$ metas de aprendizaje; MRS = metas de refuerzo social; $\mathrm{ML}=$ metas de logro; ESO = Educación Secundaria Obligatoria; ns = no significativo.

Fuente: elaboración propia. 
regresión logística binaria siguiendo el procedimiento de regresión por pasos hacia delante basado en el estadístico de Wald. Así, la variable predictora (conducta agresiva) y las variables criterio (MA, MRS y ML) fueron dicotomizadas. Para la conducta agresiva se mantuvo la misma dicotomización que para los análisis de diferencias de medias, mientras que las puntuaciones en metas académicas fueron dicotomizadas como: (a) bajas metas de aprendizaje, logro y reforzamiento social: puntuaciones iguales o inferiores al centil $25 \mathrm{y}(\mathrm{b})$ altas metas de aprendizaje, logro y reforzamiento social: puntuaciones iguales o superiores al centil 75 .

El modelado logístico permite estimar la probabilidad de que ocurra un evento, suceso o resultado (e.g., altas metas de aprendizaje) en presencia de uno o más predictores (e.g., alta conducta agresiva). Esta probabilidad es estimada mediante el estadístico denominado odd ratio (OR). Si la OR es mayor que uno el aumento de la variable independiente lleva asociado un aumento en la ventaja del suceso. Por el contrario, si la OR es menor que uno el aumento de la variable independiente conlleva una disminución de la ventaja del suceso (De Maris, 2003).

\section{Resultados}

\section{Metas académicas de estudiantes con baja y alta conducta agresiva}

La Tabla 1 presenta las diferencias entre estudiantes con alta y baja conducta agresiva en MA, MRS y ML en función del sexo y el curso académico. Los resultados indican que, tanto en ambos sexos como en todos los cursos de ESO, únicamente se encuentran diferencias estadísticamente significativas en las metas de reforzamiento social. Concretamente, en todas las muestras analizadas, las metas de reforzamiento social fueron significativamente más altas en los estudiantes con alta conducta agresiva que en los estudiantes con baja conducta agresiva. Sin embargo, el tamaño del efecto de las diferencias halladas fue pequeño en todos los casos.

\section{La conducta agresiva como predictora de las metas académicas}

Teniendo en cuenta los resultados obtenidos en los análisis de diferencias entre medias, es razonable esperar que la alta conducta agresiva no pronostique

TABLA 2

Regresión logística para la probabilidad de altas metas de reforzamiento social

\begin{tabular}{|c|c|c|c|c|c|c|c|}
\hline & & $B$ & ET & Wald & $p$ & OR & IC 95\% \\
\hline \multicolumn{8}{|l|}{ Hombres } \\
\hline MRS & Alta conducta agresiva & 0.56 & 0.14 & 16.82 & 0.00 & 1.75 & $1.34-2.28$ \\
\hline Mujeres & & & & & & & \\
\hline $\begin{array}{l}\text { MRS } \\
1^{\circ} \text { ESO }\end{array}$ & Alta conducta agresiva & 0.51 & 0.13 & 14.37 & 0.00 & 1.66 & $1.28-2.17$ \\
\hline $\begin{array}{l}\text { MRS } \\
2^{\circ} \mathrm{ESO}\end{array}$ & Alta conducta agresiva & 0.37 & 0.17 & 4.78 & 0.00 & 1.45 & $1.04-2.02$ \\
\hline $\begin{array}{l}\text { MRS } \\
\text { 3o ESO }\end{array}$ & Alta conducta agresiva & 0.51 & 0.19 & 7.63 & 0.01 & 1.67 & $1.16-2.40$ \\
\hline MRS & Alta conducta agresiva & 0.70 & 0.17 & 16.05 & 0.00 & 2.02 & $1.43-2.84$ \\
\hline & Constante & -0.76 & 0.26 & 8.32 & 0.00 & 0.47 & \\
\hline $4^{\mathrm{o}} \mathrm{ESO}$ & & & & & & & \\
\hline MRS & Alta conducta agresiva & 0.59 & 0.21 & 8.11 & 0.00 & 1.81 & $1.20-2.72$ \\
\hline
\end{tabular}

Nota. $\mathrm{B}=$ coeficiente; $\mathrm{ET}=$ error estándar; $g l=$ grados de libertad; $p=$ probabilidad; $\mathrm{OR}=$ odd ratio; $\mathrm{IC}=$ intervalo de confianza al 95\%; MRS = metas de reforzamiento social; ESO = Educación Secundaria Obligatoria; ns = no significativo.

Fuente: elaboración propia. 
las metas de aprendizaje y las metas de logro. Por tanto, los análisis de regresión logística binaria solo fueron realizados para examinar la capacidad predictiva de la alta conducta agresiva sobre las altas metas de reforzamiento social (Tabla 2).

Los resultados muestran que la proporción de casos clasificados correctamente por los modelos logísticos, en función del sexo y el curso académico, fue: $64.8 \%$ (hombres), $60.7 \%$ (mujeres), $60 \%$

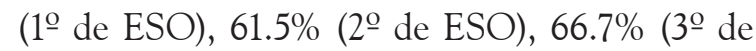
$\mathrm{ESO}$ ) y $64.4 \%$ (4⿳⺈ de ESO). La $R^{2}$ de Nagelkerke osciló entre el $4.3 \%$ ( $1^{\circ}$ ESO) y el 14.4\% ( 3 ํ ESO). Además, la Tabla 2 muestra que las odd ratio (OR) obtenidas oscilaron entre 2.02 ( $\left.3^{\circ} \mathrm{ESO}\right)$ y $1.45\left(1^{\circ} \mathrm{o}\right.$ ESO), indicando que la probabilidad de presentar altas metas de reforzamiento social es 1.75 (hombres), 1.66 (mujeres), 1.45 ( $\left.1^{\circ} \mathrm{ESO}\right), 1.67$ (2으르), $2.62\left(3^{\circ} \mathrm{ESO}\right)$ y 1.81 ( $\left.4^{\circ} \mathrm{ESO}\right)$ veces mayor en los estudiantes que presentan alta conducta agresiva que en los que presentan baja conducta agresiva. Por tanto, atendiendo a la dicotomización alta-baja conducta agresiva, la probabilidad de que los hombres, las mujeres y los estudiantes de $1^{\circ}, 2^{\circ}, 3^{\circ}$ y $4^{\circ}$ de ESO presenten altas metas de reforzamiento social cuando muestran puntuaciones altas en conducta agresiva es un $64 \%, 62 \%, 59 \%, 63 \%, 67 \%$ y $64 \%$, respectivamente, mientras que esta probabilidad es del 36\%, 38\%, 41\%, 37\%, 33\% y 36\%, respectivamente, cuando los estudiantes muestran puntuaciones bajas en conducta agresiva (véase Tabla 2).

\section{Discusión}

El presente estudio tuvo un doble objetivo. En primer lugar, analizar las diferencias en metas de aprendizaje, metas de logro y metas de reforzamiento social entre estudiantes con baja y alta conducta agresiva y, en segundo lugar, examinar la capacidad predictiva de la conducta agresiva sobre las metas académicas en una muestra representativa de estudiantes españoles de ESO.

A diferencia de los resultados hallados en investigaciones previas (e.g., Gilman \& Anderman, 2006; Little \& Garber, 2004; Wentzel \& Asher, 1995), los datos derivados de las pruebas de significación estadística aplicadas en este estudio no permiten apoyar la primera hipótesis, ya que no se encontraron diferencias estadísticamente significativas entre estudiantes con alta y baja conducta agresiva en metas de aprendizaje y metas de logro. Así, los estudiantes con alta conducta agresiva hacia sus iguales están tan interesados como sus compañeros con baja conducta agresiva por la adquisición de conocimientos, así como por conseguir buenos resultados académicos y avanzar en sus estudios. En cualquier caso, estos resultados son consistentes y complementarios con los hallados por Inglés, Martínez-González, Valle, García-Fernández y Ruiz-Esteban (2011), quienes indicaron que los estudiantes con alta conducta prosocial, de ambos sexos y de todos los cursos de ESO, presentan metas de aprendizaje y logro significativamente más altas que los estudiantes con baja conducta prosocial.

De acuerdo con la segunda hipótesis y, por tanto, con la evidencia empírica previa hallada en muestras de estudiantes de Educación Secundaria, norteamericanos (Ryan \& Shim, 2008) y españoles (López-Romero \& Romero, 2010), los estudiantes con alta conducta agresiva estudian más frecuentemente que sus compañeros con baja conducta agresiva, con el fin de conseguir juicios positivos de sus iguales, profesores y padres, evitando, simultáneamente, valoraciones negativas de los mismos (metas de reforzamiento social). Así, los estudiantes con alta conducta agresiva puntúan ligeramente más alto que sus iguales con baja conducta agresiva en ítems del AGTQ tales como, por ejemplo, "estudio porque quiero que la gente vea lo listo/a que soy" o "estudio porque me gusta sacar mejores notas que mis compañeros". Este resultado también es complementario al hallado por Inglés et al. (2011), según el cual, los estudiantes con alta y baja conducta prosocial no se diferencian en cuanto a sus metas de reforzamiento social.

La tercera hipótesis de este estudio postulaba que la conducta agresiva actuaría como un predictor negativo y estadísticamente significativo de las metas de aprendizaje y de rendimiento y como un predictor positivo y estadísticamente significativo de las metas de reforzamiento social. Sin embargo, teniendo en cuenta los resultados obtenidos en los análisis de diferencias de medias, las regresiones 
logísticas solo fueron realizadas utilizando como variable criterio las metas de reforzamiento social. Los resultados confirmaron que altas puntuaciones en conducta agresiva pronostican positiva y significativamente altas puntuaciones en metas de reforzamiento social, tanto en hombres y mujeres como en estudiantes de todos los cursos de ESO. Estos resultados son consistentes con los obtenidos en investigaciones previas (López-Romero \& Romero, 2010; Ryan \& Shim, 2008) y complementarios con los encontrados por Inglés et al. (2011), en tanto que la conducta prosocial resultó un predictor positivo y estadísticamente significativo de las metas de aprendizaje y logro pero no permitió pronosticar las metas de reforzamiento social.

En resumen, los estudiantes que se comportan agresivamente estudian fundamentalmente con el fin de evitar que los demás los valoren de forma negativa, ya que les preocupa bastante su propia imagen personal, mientras que los motivos para aprender más y avanzar en los estudios no diferencian a estudiantes con alta y baja conducta agresiva. Este patrón de resultados contrasta con el hallado en estudiantes prosociales de ESO, puesto que estos estudian principalmente para aprender más y avanzar en los estudios y no tanto para evitar que iguales, profesores y/o padres los valoren negativamente (Inglés et al., 2011).

El presente estudio presenta algunas limitaciones que futuras investigaciones deberían tener en cuenta. En primer lugar, aunque el muestreo empleado en este trabajo garantiza la representatividad de la muestra, esta se compone únicamente de estudiantes de ESO, por lo que los resultados obtenidos en el mismo no pueden generalizarse a estudiantes de otros niveles educativos como Educación Infantil, Educación Primaria, Bachillerato y Educación Superior. Futuros trabajos deberían confirmar si los resultados hallados en ESO difieren o se mantienen en otros niveles educativos con el fin de incrementar la validez externa de estos hallazgos.

En segundo lugar, el cuestionario utilizado en este estudio para la evaluación de la conducta agresiva no tiene en cuenta las diferentes dimensiones o tipos que la conforman (véase para una revisión, Torregrosa, Inglés, Estévez, Musitu \& García-Fer- nández, 2011). Por tanto, futuros estudios deberían evaluar los distintos tipos de comportamiento agresivo y analizar la relación que estos mantienen con las metas académicas.

En tercer lugar, el uso del AGTQ como medida de las metas académicas no diferencia entre tendencias de aproximación y de evitación como dimensiones independientes, tanto en el caso de las metas de aprendizaje como en el de las metas de rendimiento (Pintrich, 2003), aún cuando la tendencia actual en investigación motivacional hace especial énfasis en esta distinción (e.g., Valle, Rodríguez et al., 2009). Por ello, sería conveniente que futuras investigaciones analicen cómo se relaciona la conducta agresiva con estos dos tipos de tendencias motivacionales. Finalmente, es importante resaltar que en esta investigación se han empleado únicamente medidas de autoinforme, lo cual puede introducir sesgos derivados de la deseabilidad social, aspecto este particularmente importante cuando se evalúa la conducta agresiva, puesto que los adolescentes tienden a infraestimarla (e.g., Dodge et al., 2006). En este sentido, sería recomendable que futuros estudios utilicen diferentes procedimientos de evaluación tales como, por ejemplo, nominaciones sociométricas y escalas de valoración por los iguales y profesores.

A pesar de estas limitaciones y consideraciones, el presente estudio puede considerarse pionero, ya que ha demostrado la existencia de una relación positiva y estadísticamente significativa entre conducta agresiva autoinformada y metas académicas en una muestra de estudiantes españoles de ESO.

Finalmente, los resultados del presente estudio sugieren algunas reflexiones tanto para los profesores, como para los profesionales e investigadores de la Psicología y la Educación. Por ejemplo, las sucesivas reformas educativas realizadas durante las últimas décadas se han centrado en la motivación académica y en las calificaciones escolares de los estudiantes de ESO, sin profundizar en el conocimiento relativo a la influencia que pueden tener diversos factores sociales o contextuales sobre las mismas. En este sentido, los resultados de este estudio han demostrado la existencia de relaciones entre el comportamiento agresivo y la motivación 
escolar. Estos resultados confirman lo argumentado por otros autores, quienes indican que la comunidad educativa debería seguir enfatizando sobre diversas variables intrapersonales (como la motivación escolar y el rendimiento académico), pero también atender a los factores interpersonales, por ejemplo, mediante el establecimiento de relaciones cooperativas o prosociales entre los alumnos de un aula (Wentzel, 2005; Wentzel \& Watkins, 2002). En esta línea, Inglés et al. (2008) indicaron que los responsables de políticas educativas y, en última instancia, las escuelas y los educadores, deberían considerar la elevada prevalencia de estudiantes prosociales como un recurso preventivo y terapéutico para promover el aprendizaje escolar y mejorar las relaciones interpersonales de los estudiantes con problemas de comportamiento agresivo. La investigación avala esta propuesta, demostrando, consistentemente, que el modelado de conductas sociales positivas realizado por iguales socialmente hábiles y prosociales es una de las técnicas de intervención cognitivo-conductuales que, junto al efecto de otras técnicas, mejoran sustancialmente la eficacia del entrenamiento en habilidades sociales aplicado a estudiantes de Educación Secundaria con comportamientos agresivos (e.g., Beelman \& Lösel, 2006; Sukhodolsky, Kassinove \& Gorman, 2004) y a estudiantes con y sin problemas en su funcionamiento social (Rosa et al., 2002).

\section{Referencias}

Alderman, M. K. (2008). Motivation for achievement: Possibilities for teaching and learning (3. - ed.). New York: Taylor \& Francis.

Andreu, J. M., Peña, M. E. \& Larroy, C. (2010). Conducta antisocial, impulsividad y creencias justificativas: análisis de sus interrelaciones con la agresión proactiva y reactiva en adolescentes. Behavioral Psychology/Psicología Conductual, 18(1), 52-72.

Ang, R. P. (2007). Factor structure of the 12-item Aggression Questionnaire: Further evidence from Asian adolescent samples. Journal of Adolescence, 30(4), 671-685.

Beelman, A. \& Lösel, F. (2006). Child social skills training in development crime prevention: Effects on antisocial behavior and social competence. Psicothema, 18(3), 603-610.

Broc, M. A. (2006). Motivación y rendimiento académico en alumnos de Educación Secundaria Obligatoria y Bachillerato LOGSE. Revista de Educación, 340, 379-414.

Butler, R. (2006). Are mastery and ability goals both adaptive? Evaluation, initial goal construction and the quality of task engagement. British Journal of Educational Psychology, 76(3), 595-611.

Castillo, I., Balaguer, I. \& Duda, J. L. (2001). Perspectivas de meta de los adolescentes en el contexto académico. Psicothema, 13(1), 79-86.

Cohen, J. (1988). Statistical power analysis for the behavioural sciences (2. ${ }^{\text {a }}$ ed.). Hillsdale, NJ: Erlbaum.

Covington, M. V. (2000). Goal theory, motivation and school achievement: An integrative review. Annual Reviews of Psychology, 51, 171-200.

Delgado, B., Inglés, C. J., García-Fernández, J. M., Castejón, J. L. \& Valle, A. (2010). Diferencias de género y curso en metas académicas en alumnos de Educación Secundaria Obligatoria. Revista Española de Pedagogía, 68(245), 67-84.

De Maris, A. (2003). Logistic regression. En J. A. Schinka \& W. F. Velicer (Eds.), Research methods in psychology (pp. 509-532). New Jersey: John Wiley \& Sons.

Díaz-Aguado, M. J., Martínez-Arias, R. \& MartínSeoane, G. (2004). En Prevención de la violencia y la lucha contra la exclusión desde la adolescencia (pp. 21-88). Madrid: Ministerio de Trabajo y Asuntos Sociales, Instituto de la Juventud.

Dina, F. \& Efklides, A. (2009). Student profiles of achievement goals, goal instruction and external feed-back: Their effect on mathematical task performance and affect. European Journal of Education and Psychology, 2(3), 235-262.

Dodge, K. A., Coie, J. D. \& Lynam, D. (2006). Aggression and antisocial behaviour in youth. En N. Eisenberg, W. Damon \& R. M. Lerner (Eds.), Handbook of child psychology. Social, emotional, and personality development (Vol. 3, pp. 719-788). Hoboken, NJ: John Wiley \& Sons.

Dweck, C. S. (1986). Motivational processes affecting learning. American Psychologist, 41(19), 1040-1048. 
Elliot, A. \& Dweck, C. (2005). Handbook of competence and motivation. Nueva York: Guilford.

Estévez, E., Martínez, B., Herrero, J. \& Musitu, G. (2006). Aggressive and non-aggressive rejected students: An analysis of their differences. Psychology in the Schools, 43(3), 387-400.

Estévez, E., Martínez, B. \& Musitu, G. (2006). La autoestima en adolescentes agresores y víctimas en la escuela: la perspectiva multidimensional. Intervención Psicosocial, 15(2), 223-232.

Estévez, E., Murgui, S., Moreno, D. \& Musitu, G. (2007). Estilos de comunicación familiar, actitud hacia la autoridad institucional y conducta violenta del adolescente en la escuela. Psicothema, 19(1), 108-113.

Estévez, E., Murgui, S. \& Musitu, G. (2008). Psychosocial adjustment in aggressors, pure victims and aggressive victims at school. European Journal of Education and Psychology, 1(2), 33-44.

Farmer, T. W., Estell, D. B., Leung, M. -C., Trott, H., Bishop, J. \& Cairns, B. D. (2003). Individual characteristics, early adolescent peer affiliations, and school dropout: An examination of aggressive and popular group types. Journal of School Psychology, 41(3), 217-232.

Gilman, R. \& Anderman, E. M. (2006). The relationship between relative levels of motivation and intrapersonal, interpersonal, and academic functioning among older adolescents. Journal of School Psychology, 44(5), 375-391.

Hayamizu, T. \& Weiner, B. (1991). A test of Dweck's model of achievement goals as related to perceptions of ability. Journal of Experiemental Education, 59(3), 226-234.

Herrenkohl, T. I., Catalano, R. F., Hemphill, S. A. \& Toumbourou, J. W. (2009). Longitudinal examination of physical and relational aggression as precursors to later problem behaviors in adolescents. Violence and Victims, 24(1), 3-19.

Herrero, J., Estévez, E. \& Musitu, G. (2006). The relationships of adolescent school-related deviant behaviour and victimization with psychological distress: Testing a general model of the mediational role of parents and teachers across groups of gender and age. Journal of Adolescence, 29(5), 671-690.
Inderbitzen, H. M. \& Foster, S. L. (1992). The Teenage Inventory of Social Skills: Development, reliability, and validity. Psychological Assessment, 4(4), 451-459.

Inglés, C. J., Delgado, B., Bautista, R., Torregrosa, M. S., Espada, J. P., García-Fernández, J. M., et al. (2007). Factores psicosociales relacionados con el consumo de alcohol y tabaco en adolescentes españoles. International Journal of Clinical and Health Psychology, 7(2), 403-420.

Inglés, C. J., Delgado, B., García-Fernández, J. M., Ruiz-Esteban, C. \& Díaz-Herrero, A. (2010). Sociometric types and social interaction styles in a sample of Spanish adolescents. Spanish Journal of Psychology, 13(2), 728-738.

Inglés, C. J., García-Fernández, J. M., Castejón, J. L., Valle, A., Delgado, B. \& Marzo, J. C. (2009). Reliability and validity evidence of scores on the achievement goal tendencies questionnaire in a sample of Spanish students of compulsory secondary education. Psychology in the Schools, 46(10), 1048-1060.

Inglés, C. J., Hidalgo, M. D., Méndez, F. X. \& Inderbitzen, H. M. (2003). The Teenage Inventory of Social Skills: Reliability and validity of the Spanish translation. Journal of Adolescence, 26(4), 505-510.

Inglés, C. J., Martínez-González, A. E., Valle, A., García-Fernández, J. M. \& Ruíz-Esteban, C. (2011). Conducta prosocial y motivación académica en estudiantes españoles de Educación Secundaria Obligatoria. Universitas Psychologica, 10(2), 451465.

Inglés, C. J., Martínez-Monteagudo, M. C., Delgado, B., Torregrosa, M.S., Redondo, J., Benavides, G., et al. (2008). Prevalencia de la conducta agresiva, conducta prosocial y ansiedad social en una muestra de adolescentes españoles: Un estudio comparativo. Infancia y Aprendizaje, 31(4), 449-461.

Inglés, C. J., Marzo, J. C., Castejón, J. L., Núñez, J. C., Valle, A., García-Fernández, J. M., et al. (2011). Factorial invariance and latent mean differences of scores on the Achievement Goal Tendencies Questionnaire across gender and age in a sample of Spanish students. Learning and Individual Differences, 21(1), 138-143. 
Inglés, C. J., Méndez, F. X., Hidalgo, M. D., Rosa, A. I. \& Estévez, C. (2003). Evaluación de las habilidades sociales en educación secundaria: revisión de cuestionarios, inventarios y escalas. Psicología Educativa, 9(2), 71-87.

Jimerson, S. R. \& Ferguson, P. (2007). A longitudinal study of grade retention: Academic and behavioral outcomes of retained students through adolescence. School Psychology Quarterly, 22(3), 314-339.

Lahey, B. B., Schwab-Stone, M., Goodman, S. H., Waldman, I. D., Canino, G., Rathouz, P. J., et al. (2000). Age and gender differences in oppositional behavior and conduct problems: A cross-sectional household study of middle childhood and adolescence. Journal of Abnormal Psychology, 109(3), 488-503.

Little, S. \& Garber, J. (2004). Interpersonal and achievement orientations and specific stressors predict depressive and aggressive symptoms. Journal of Adolescent Research, 19(1), 63-84.

López-Romero, L. \& Romero, E. (2010). Goals during adolescence and their relationship with antisocial behavior. Spanish Journal of Psychology, 13(1), $166-177$.

Loveland, J. M., Lounsbury, J. W., Welsh, D. \& Buboltz, W. (2007). The validity of physical aggression in predicting adolescent academic performance. British Journal of Educational Psychology, 77(1), 167-176.

Marcus, R. F. (2007). Aggression and violence in adolescence. New York: Cambridge University Press.

Marsh, H. W., Parada, R. H. \& Ayotte, V. (2004). A multidimensional perspective of relations between self-concept (Self-Concept Questionnaire II) and adolescent mental health (Youth Self-Report). Psychological Assessment, 16(1), 27-41.

Massey, E. K., Gebhardt, W. A. \& Garnefski, N. (2008). Adolescent goal content and pursuit: A review of the literature from the past 16 years. Developmental Review, 28(4), 421-460.

Meece, J. L., Anderman, E. L. \& Anderman, L. H. (2006). Classroom goal structure, student motivation, and academic achievement. Annual Review of Psychology, 57, 487-503.

Ministerio de Educación y Ciencia. (2010). Las cifras de la educación en España. Estadísticas e indicadores. Recuperado el 14 de febrero de 2011, de http:// www.educacion.es/mecd/estadisticas/educativas/ cee/Edicion2011/D3.pdf

Núñez, J. L., Martín-Albo, J., Navarro, J. G. \& Suárez, Z. (2010). Adaptación y validación de la versión española de la Escala de Motivación Educativa en estudiantes de educación secundaria postobligatoria. Estudios de Psicología, 31(1), 89-100.

Peets, K. \& Kikas, E. (2006). Aggressive strategies and victimization during adolescence: Grade and gender differences, and cross-informant agreement. Aggressive Behavior, 32(1), 68-79.

Pintrich, P. R. (2003). Motivation and classroom learning. En Reynolds \& Miller (Eds.), Handbook of psychology (pp. 103-122). Hoboken, NJ: Wiley \& Sons.

Rodríguez-Mayoral, J. M., Martínez-Arias, R., DíazAguado, M. J. \& Morentín, R. (2008). Comportamiento violento en adolescentes: su relación con las estrategias cognitivas y el rendimiento académico. Psicología Educativa, 14(1), 63-81.

Rosa, A. I., Inglés, C. J., Olivares, J., Espada, J. P., Sánchez-Meca, J. \& Méndez, F. X. (2002). Eficacia del entrenamiento en habilidades sociales con adolescentes: de menos a más. Behavioral Psychology/ Psicología Conductual, 10(3), 543-561.

Ryan, A. M. \& Shim, S. S. (2008). An exploration of young adolescents' social achievement goals and social adjustment in middle school. Journal of Educational Psychology, 100(3), 672-687.

Saab, H. \& Klinger, D. (2010). School differences in adolescent health and wellbeing: Findings from the Canadian health behavior in school-aged children study. Social Science \& Medicine, 70(6), 850-858.

Santisteban, C., Alvarado, J. M. \& Recio, P. (2007). Evaluation of a Spanish version of the Buss and Perry Aggression Questionnaire: Some personal and situational factors related to aggression scores of young subjects. Personality and Individual Differences, 42(8), 1453-1465.

Steinmayr, R. \& Spinath, B. (2009). The importance of motivation as a predictor of school achievement. Learning and Individual Differences, 19(1), 80-90.

Sukhodolsky, D. G., Kassinove, H. \& Gorman, B. S. (2004). Cognitive-behavioral therapy for anger in children and adolescents: A meta-analysis. Aggression and Violent Behavior, 9(3), 247-269. 
Sun, S., Pan, W. \& Wang, L. L. (2010). A comprehensive review of effect size reporting and interpreting practices in academic journals in education and psychology. Journal of Educational Psychology, 102(4), 989-1004.

Toldos, M. P. (2005). Sex and age differences in selfestimated physical, verbal and indirect aggression in Spanish adolescents. Aggressive Behavior, 31(1), 13-23.

Torregrosa, M. S., Inglés, C. J., Delgado, B. \& GarcíaFernández, J. M. (2008, julio). Academic causal attribution in aggressive Spanish adolescents. Trabajo presentado en el XXIX International Congress of Psychology, Berlín, Alemania.

Torregrosa, M. S., Inglés, C. J., Estévez, E., Musitu, G. \& García-Fernández, J. M. (2011). Evaluación de la conducta violenta en la adolescencia: revisión de cuestionarios, inventarios y escalas en población española. Aula Abierta, 39(1), 37-50.

Torregrosa, M. S., Inglés, C. J., García-Fernández, J. M., Gázquez, J. J., Díaz-Herrero, A. \& Bermejo, R. M. (2012). Conducta agresiva entre iguales y rendimiento académico en una muestra de adolescentes españoles. Behavioral Psychology/Psicología Conductual, 20(2), 263-280.

Trianes, M. V. (2000). La violencia en contextos escolares. Málaga: Aljibe.

Valle, A., Cabanach, R. G., Núñez, J. C., GonzálezPienda, J. A., Rodríguez, S. \& Piñeiro, I. (2003). Cognitive, motivational, and volitional dimensions of learning: An empirical test of a hypothetical model. Research in Higher Education, 44(5), 557-580.
Valle, A., Núñez, J. C., Cabanach, R. G., Rodríguez, S., González-Pienda, J. A. \& Rosario, P. (2009). Perfiles motivacionales en estudiantes de Secundaria: análisis diferencial en estrategias cognitivas, estrategias de autorregulación y rendimiento académico. Revista Mexicana de Psicología, 26(1), 113-124.

Valle, A., Rodríguez, S., Cabanach, R. G., Núñez, J. C., González-Pienda, J. A. \& Rosario, P. (2009). Metas académicas: perspectiva histórica y conceptual e implicaciones educativas. Revista de Investigación Psicoeducativa, 7(3), 307-331.

Valle, A., Rodríguez, S., Cabanach, R. G., Núñez, J. C., González-Pienda, J. A. \& Rosario, P. (2010). Perfiles motivacionales y diferencias en variables afectivas, motivacionales y de logro. Universitas Psychologica, 9(1), 109-121.

Wentzel, K. R. (2005). Peer relationships, motivation, and academic performance at school. En J. Elliot \& C. S. Dweck (Eds.), Handbook of competence and motivation (pp. 279-296). New York: Guilford Press.

Wentzel, K. R. \& Asher, S. R. (1995). The academic lives of neglected, rejected, popular, and controversial children. Child Development, 66(3), 754-763.

Wentzel, K. R. \& Watkins, D. E. (2002). Peer relationships and collaborative learning as contexts for academic enablers. School Psychology Review, 31(3), 366-377.

Wigfield, A. \& Eccles, J. S. (2002). Development of achievement motivation. San Diego, CA: Academic Press. 
